\title{
Application of multivariate single-step SNP best linear unbiased predictor model and revised SNP list for genomic evaluation of dairy cattle in Australia
}

\author{
K. V. Konstantinov ${ }^{1 *}$ and M. E. Goddard ${ }^{2}$ \\ ${ }^{1}$ DataGene Limited, Agriculture Victoria, AgriBio Centre for AgriBusiness, 5 Ring Rd., Bundoora, Victoria 3083, Australia \\ ${ }^{2}$ Melbourne School of Land and Environment, University of Melbourne, Parkville, Victoria 3010, Australia
}

\begin{abstract}
The objectives of this study were (1) to evaluate the computational feasibility of the multitrait test-day single-step SNP-BLUP (ssSNP-BLUP) model using phenotypic records of genotyped and nongenotyped animals, and (2) to compare accuracies (coefficient of determination; $\mathrm{R}^{2}$ ) and bias of genomic estimated breeding values (GEBV) and de-regressed proofs as response variables in 3 Australian dairy cattle breeds (i.e., Holstein, Jersey, and Red breeds). Additive genomic random regression coefficients for milk, fat, protein yield and somatic cell score were predicted in the first, second, and third lactation. The predicted coefficients were used to derive 305-d GEBV and were compared with the traditional parent averages obtained from a BLUP model without genomic information. Cow fertility traits were evaluated from the 5-trait repeatability model (i.e., calving interval, days from calving to first service, pregnancy diagnosis, first service nonreturn rate, and lactation length). The de-regressed proofs were only for calving interval. Our results showed that ssSNP-BLUP using multitrait test-day model increased reliability and reduced bias of breeding values of young animals when compared with parent average from traditional BLUP in Australian Holsten, Jersey, and Red breeds. The use of a custom selection of approximately 46,000 SNP (custom XT SNP list) increased the reliability of GEBV compared with the results obtained using the commercial Illumina 50K chip (Illumina, San Diego, CA). The use of the second preconditioner substantially improved the convergence rate of the preconditioned conjugate gradient method, but further work is needed to improve the efficiency of the computation of the Kronecker matrix product by vector. Application of ssSNP-BLUP to multitrait random regression models is computationally feasible.
\end{abstract}

Received January 21, 2020.

Accepted April 21, 2020.

*Corresponding author: Kon.Konstantinov@agriculture.vic.gov.au
Key words: Holstein, Jersey, Australian Red, random regression, single-step SNP-BLUP

\section{INTRODUCTION}

National genomic evaluations of Australian Holstein and Jersey breeds have been published since 2010 (Nieuwhof et. al., 2010). The procedure for genomic evaluation involves the calculation of several components: (1) traditional BLUP breeding values without genomic information; (2) daughter yield deviations or de-regressed proofs (DRP); (3) direct genomic values (DGV; VanRaden, 2008; Hayes et al., 2009); (4) second EBV from pedigree within the cohort of genotyped animals used for correction of DGV; and (5) genomic enhanced breeding values (GEBV) and corresponding reliabilities obtained using formulas presented by Harris and Johnson (2010).

It has been recognized that the multistep approach for calculation of GEBV has inherent problems (Legarra et al., 2009). The existence of these problems has motivated the development of a unified procedure for prediction of genetic values that utilize phenotypic and genomic information (Legarra et al., 2009; Aguilar et al., 2010; Christensen and Lund, 2010). This procedure became known as single-step genomic BLUP (ssGBLUP). It integrates the genomic relationship matrix $\mathbf{G}$ and the pedigree relationship matrix $\mathbf{A}$ into a single matrix $\mathbf{H}$, which replaces the traditional relationship matrix A used in BLUP equations. However, sometimes $\mathbf{A}$ and $\mathbf{G}$ are not on the same scale, which requires scaling factors to blend $\mathbf{G}$ to the relationship matrix for genotyped animals A22. Several studies in the literature investigated the best combination of scaling factors in the context of evaluation of milk production traits (Misztal et al., 2013; Lourenco et al., 2014; Koivula et al., 2015; Kang et al., 2018; Oliveira et al., 2019). Legarra et al. (2015) proposed the use of pseudoindividuals called metafounders that encapsulate genetic heterozygosity and relationships within and across base pedigree populations (i.e., ancestral populations); they demonstrated that the use of meta- 
founders leads to compatibility of genomic and pedigree relationship matrices.

Computationally the ssGBLUP approach has been rated as demanding especially with large data sets and complex models such as test-day models. The performance of ssGBLUP has been evaluated on random regression test-day data (Koivula et al., 2015; Baba et al., 2017; Oliveira et al., 2019).

A different single-step approach has been proposed by Fernando et al. (2014), which requires explicit imputation of markers for nongenotyped animals and then fitting the marker effect in the model. All the unknowns were suggested to be estimated using Markov-chain Monte Carlo algorithm, which may be computationally challenging. In another study (Fernando et al., 2016), the authors presented a hybrid model featuring a considerable reduction in computing effort.

To date, most studies tended to focus on the ssGBLUP model. Liu et al. (2014, 2016) developed a singlestep SNP-BLUP model (ssSNP-BLUP) equivalent to the ssGBLUP that simultaneously estimates EBV and marker effects and does not require the construction of the genomic matrix $\mathbf{G}$ and its inverse. Osawa and Saburi (2018) applied this approach to a 305-d repeatability model in the Japanese Holstein population. To the best of our knowledge, no study has been conducted applying ssSNP-BLUP to large multivariate test-day models.

Therefore, the present study aimed to (1) investigate the computational feasibility of ssSNP-BLUP model in a multitrait scenario with large commercial data sets and (2) investigate its predictive ability in Australian Holstein, Jersey, and Red breeds.

\section{MATERIALS AND METHODS}

\section{Phenotype Data}

The phenotypes and pedigrees used in this study were extracted from the DataGene (https://datagene .com.au/) Australian national database. The data sets consisted of test-day records in the first 3 lactations for milk, fat, and protein yield, and SCS of cows from all breeds calved between 1985 and 2017. The data sets for cow fertility did not have restrictions on lactation number. The usual quality control filtering was applied as per a standard commercial run. After the quality control 2 data sets were prepared. The first data set (full data set) included all information up to July 2019. The second one (reduced data set) contained all phenotypic information available until July 2015. For each breed, we identified genotyped animals born between 2009 and 2013 in 2019 data. Bulls having 50 or more daughters in the full data set were considered as candidates for validation. For the cows, the candidates were those having at least 3 test-day records and at least one daughter with records in July 2019 data. The records of the validation cows and their daughters, as well as the records of the daughters of the validation bulls were deleted from 2015 data, but a full pedigree was retained. For simplicity, the genetic groups were removed from the pedigrees in both data sets. The reduced data set was used to predict GEBV using ssSNP-BLUP, as well as traditional BLUP EBV. The full data set was used to obtain traditional EBV and related DRP, which were used as a benchmark to validate GEBV and parent average (PA) obtained from the reduced data. Considering all breeds together, the number of animals in the pedigree and test-day records is shown in Table 1.

\section{Genotype Data}

Most bulls were genotyped with the Illumina BovineSNP50 BeadChip (50K chip, with 40,080 SNP passing quality filters; Illumina, San Diego, CA) or the Illumina BovineHD BeadChip (HD, with 632,003 SNP passing quality filters). Most cows were genotyped with lower density custom SNP arrays with approximately 8,800 SNP of which 8,056 overlapped the Illumina BovineSNP50 BeadChip. Animals with lower density genotypes were first imputed to the Illumina $50 \mathrm{~K}$ chip and subsequently all $50 \mathrm{~K}$ genotypes were imputed to

Table 1. Number of animals and records used in the analyses in both reduced and full data sets

\begin{tabular}{|c|c|c|c|c|c|c|}
\hline \multirow[b]{2}{*}{ Trait } & \multicolumn{3}{|c|}{ Reduced data set (up to 2015) } & \multicolumn{3}{|c|}{ Full data set (up to 2019) } \\
\hline & Pedigree & $\begin{array}{l}\text { Number of cows } \\
\text { with records }\end{array}$ & $\begin{array}{c}\text { Number of } \\
\text { test-day records }\end{array}$ & Pedigree & $\begin{array}{l}\text { Number of cows } \\
\text { with records }\end{array}$ & $\begin{array}{c}\text { Number of } \\
\text { test-day records }\end{array}$ \\
\hline $\mathrm{MY}, \mathrm{FY}, \mathrm{PY}^{2}$ & $5,595,365$ & $5,008,025$ & $40,025,131$ & $6,051,806$ & $5,510,437$ & $42,751,873$ \\
\hline SCS & $4,594,577$ & $3,823,066$ & $29,055,127$ & $5,055,957$ & $4,311,060$ & $31,782,604$ \\
\hline Cow fertility & $2,407,400$ & $1,647,559$ & $3,981,702$ & $2,880,316$ & $2,124,471$ & $5,109,517$ \\
\hline
\end{tabular}

${ }^{1}$ For cow fertility, number of repeated records.

${ }^{2} \mathrm{MY}=$ milk yield $; \mathrm{FY}=$ fat yield; and $\mathrm{PY}=$ protein yield. 
HD. Next, the custom SNP from the lower density panels were added back, or imputed, into HD genotypes and all animals were imputed to whole-genome sequence using an imputation reference that comprised 2,333 individual sequences from run 6 of the 1000 Bull Genomes Project (Daetwyler et al., 2014; Bouwman et al., 2018).

Raw genotypes were filtered using the GenCall score, such that any SNP with less than $90 \%$ of their GenCall scores $>0.6$ were removed, and animals with less than $90 \%$ of their SNP genotypes with GenCall score $>0.6$ were also removed. The few remaining SNP calls with GenCall score $<0.6$ were set to zero and imputed with Fimpute. Animals were also removed if their genotypes showed excessive heterozygosity $(>0.4)$. Imputation up to HD was undertaken with Fimpute (Sargolzaei et al., 2014), whereas for imputation to sequence, the HD and sequence genotypes were first phased using Eagle (Version 2.3; Loh et al., 2016) and then imputed using Minimac3 (Das et al., 2016). Any imputed sequence variant with a Minimac $\mathrm{R}^{2}$ of less than 0.4 was discarded.

Two quite different genotype sets were used in this study: the 50K chip (Illumina BovineSNP50 BeadChip) with 40,080 SNP and a custom XT SNP list with 46,516 variants expected to be enriched for causative mutations or markers in strong linkage disequilibrium with causal variants for one or more of 34 important dairy traits (Xiang et al, 2019). The variants included all on the lower density SNP panel used for cows, 6,226 that overlapped the $50 \mathrm{~K}$ and/or HD panels, while the remaining 31,481 were sequence variants. Animal genotypes for the custom XT SNP list were generated from the imputed sequence data described above. This SNP list had been pre-filtered using the Illumina DesignStudio Software (https://sapac.illumina.com/informatics/ sample-experiment-management/custom-assay-design .html) to include only variants with design score $>0.4$. It was also designed to provide relatively even genomewide coverage.

Table 2 shows the total number of genotyped animals for each breed.

Table 2. Number of genotyped animals by breed

\begin{tabular}{lrrr}
\hline & \multicolumn{3}{c}{ Breed } \\
\cline { 2 - 4 } Item & Holstein & Jersey & $\begin{array}{c}\text { Red } \\
\text { breeds }\end{array}$ \\
\hline All animals & & & \\
$\quad$ Bulls & 5,013 & 1,278 & 350 \\
Cows & 53,397 & 10,617 & 3,233 \\
Validation animals & & & 77 \\
$\quad$ Bulls & 691 & 158 & 898 \\
Cows & 5,941 & 1,939 & \\
\hline
\end{tabular}

\section{Statistical Analyses}

The models for production traits and SCS used in this study were similar to the multivariate random regression models used for routine genetic evaluation by DataGene in Australia. In matrix notation, the general model can be described as follows:

$$
\mathbf{y}=\mathbf{X} \boldsymbol{\delta}+\mathbf{Z u}+\mathbf{W} \mathbf{p}+\mathbf{e}
$$

where $\mathbf{y}$ is a vector containing phenotypic records for all breeds, $\boldsymbol{\delta}$ is a vector of fixed effects, $\mathbf{u}$ is a vector containing random regression coefficients for animal additive genetic effects, $\mathbf{p}$ is a vector of random regression coefficients for permanent environmental effects, and $\mathbf{e}$ is a vector of random residuals. The matrices $\mathbf{X}$, $\mathbf{Z}$, and $\mathbf{W}$ are incidence matrices relating observations to factors. A detailed description of the models and underlying assumptions is presented in Konstantinov et al. (2009, 2015) and Haile-Mariam and Pryce (2015). Appending a vector of SNP effects $g$ to $u$ in model [1], Liu et al. (2014, 2016) developed an equivalent to ssGBLUP single-step SNP model (ssSNP-BLUP). Originally derived as univariate model, it can be easily extended to a multivariate case. The linear system of a multivariate mixed model equations of ssSNP-BLUP can be written as in system [2].

In system [2], $\mathbf{S}$ is an $\mathrm{n} \times \mathrm{m}$ matrix, where $\mathrm{n}=$ number of genotyped animals and $\mathrm{m}=\mathrm{SNP}$ markers; 2 $-2 p_{j}, 1-2 p_{j},-2 p_{j}$, for genotype AA, Aa, or aa of the $j$ th marker (VanRaden, 2008), $p_{j}$ represents the allele frequency of the $j$ th SNP marker. Under the assumption of uncorrelated SNP effects, $\mathbf{B}$ is a $\mathrm{m} \times \mathrm{m}$ diagonal matrix containing the variance(s) of $\mathbf{g}$; $\mathbf{R}$ is the residual co-variance matrix, $\mathbf{A}$ is the pedigree relationship matrix, and $\mathbf{I}$ is an identity matrix; and $\mathbf{u} \mathbf{1}$ and $\mathbf{u} \mathbf{2}$ are vectors for nongenotyped and genotyped animals, respectively. The residual polygenic variance parameter $k$ may take values between 0 and 1 but excluding boundary values (Liu et al., 2014). The matrices $\mathbf{G}_{0}^{-1}$ and $\mathbf{G}_{1}^{-1}$ are the inverses of the additive genetic and permanent environmental co-variance matrices, respectively.

System [2] can be solved by different computing strategies. One way to solve it is to separate SNP effects from the rest and iterate between the 2 systems by using the successive over-relaxation, successive underrelaxation technique (Legarra and Ducrocq, 2012; Liu et al., 2014). A similar approach has been used by Osawa and Saburi (2018). We solved the linear systems of equations of ssSNP-BLUP without splitting it by the preconditioned conjugate gradient (PCG) (Barrett et al., 1994) method using the in-house BLUP $\mathrm{C}++$ program (Konstantinov et al., 2009), modified to accom- 


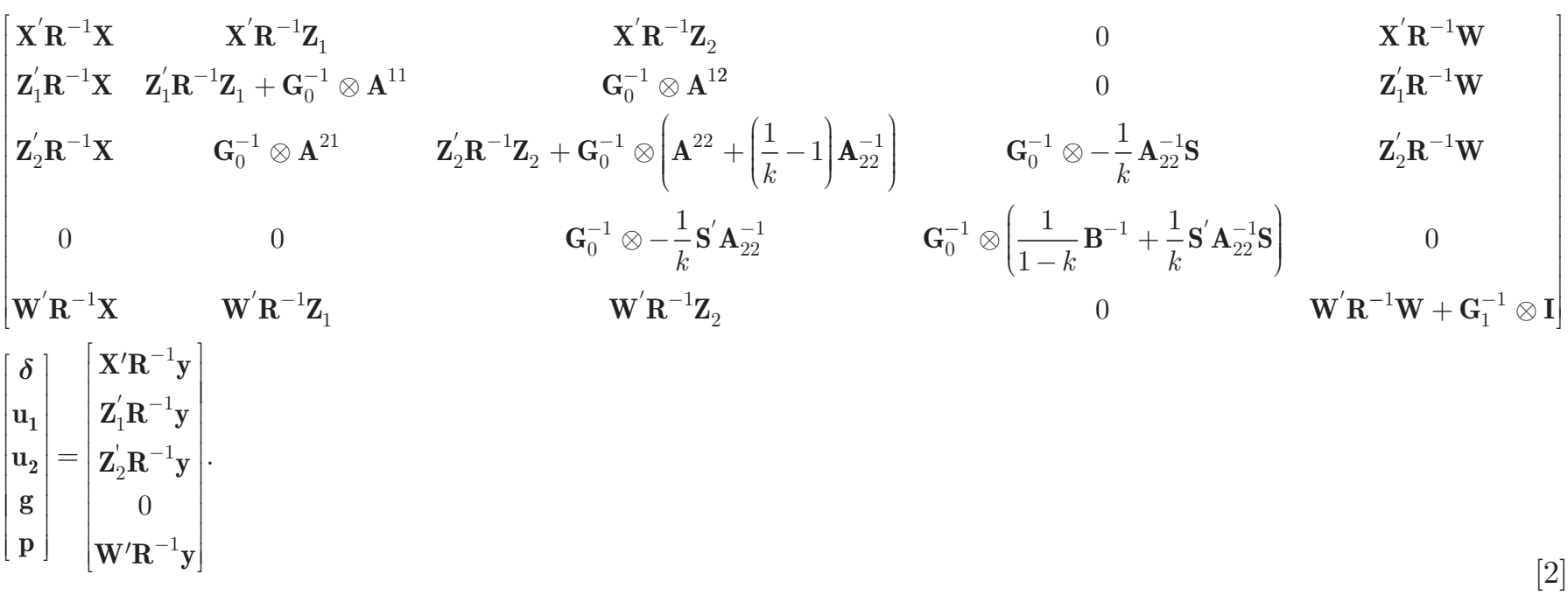

modate genomics, and exploiting basic linear algebra subprograms (BLAS) and sparse BLAS routines and the parallel direct sparse solver PARDISO, all from the multithreaded Intel Math Kernel Library 19.0.1.144, and OpenMP parallel computing.

\section{Calculation of A22 and Kronecker Product Matrix by Vector}

The system of ssSNP-BLUP equations require computing the inverse of the relationship matrix A22 for the genotyped animals. Its inverse was obtained as follows: for each breed, Holstein, Jersey, and Red, a vector containing the national ID of genotyped animals was created. For those animals all relatives were extracted from the master pedigree, thus creating a new pedigree for each breed. The newly created pedigrees were used for calculation of the inverses of the relationship matrices for the 3 breeds. The numerator relationship matrices were calculated as $\mathbf{T D} \mathbf{T}^{\prime}$, where $\mathbf{D}$ is a diagonal matrix containing the variance for Mendelian sampling. The inbreeding coefficients required for the construction of $\mathbf{D}$ were obtained using the method of Sargolzaei et al. (2005). The inverses were stored in sparse matrix format. The vectors of ID were used to create permutation matrices needed for the rearrangement of genotyped and nongenotyped animals. Given the permutation matrix $\mathbf{P}$ of $\mathrm{N} \times \mathrm{M}$ for the rows and the permutation matrix $\mathbf{Q}$ of $\mathrm{M} \times \mathrm{N}$ for the columns we wish to compute $\mathbf{P} A^{-1} \mathbf{Q}^{\prime}=\left[\mathbf{Q}\left(\mathbf{P A}^{-1}\right)^{\prime}\right]^{\prime}$ (Saad, 2003). This procedure is quite fast because each row of the permutation matrix contains one element equal to 1 , the remaining elements of the row being equal to 0 . This way we created $\mathbf{A}^{-1}$ partitioned as $\left[\begin{array}{ll}\mathbf{A}_{11} & \mathbf{A}_{12} \\ \mathbf{A}_{21} & \mathbf{A}_{22}\end{array}\right]^{-1}$. The re- quired matrix $\mathbf{A}_{22}^{-1}$ was calculated as a Schur complement (Saad, 2003) using publicly available sparse matrix library PARDISO.

It should be pointed out that there are algorithms that do not require calculations of the inverse of $\mathbf{A 2 2}$ (Masuda et al., 2017; Strandén et al., 2017). Solving system [2] by PCG requires the calculation of 4 Kronecker products. This could be quite a challenging task given that the Kronecker products involve dense matrices. The calculation of the product $\mathbf{G}_{0}^{-1} \otimes \mathbf{A}^{11}$ by vector is relatively fast because $\mathbf{A}^{11}$ contains only 3 elements per row and usually is performed in 3 parts, that is, $\mathrm{Bq}$ $=\mathbf{G}_{0}^{-1} \otimes\left[\mathbf{A}^{11}\right] p$, while traversing the pedigree. The multiplication of Kronecker product matrix by vector can be accelerated with the use of the so-called vec trick. Henderson and Searle (1981) gave a comprehensive review on the subject. Vec trick: Let $\mathbf{A} \in \Re^{\text {cxd }}, \mathbf{X} \in \Re^{\text {bxc }}$, and $\mathbf{B} \in \Re^{\text {axb }}$ be matrices. Then, $\left(\mathbf{A}^{\mathbf{T}} \otimes \mathbf{B}\right) \operatorname{vec}(\mathbf{X})=\operatorname{vec}(\mathbf{B X A})$. Obviously, the righthand side is considerably faster to compute than the left-hand side because the direct computation of a large Kronecker product is avoided. The product $\mathbf{B X}$ was never computed explicitly, instead $\mathbf{y}=\mathbf{B} \mathbf{x}$ was calculated for each row of $\mathbf{X}$ (where $\mathbf{x}$ is a row of $\mathbf{X}$ ) using DGEMV from LAPACK, and then all elements of $\mathbf{y}$ were multiplied by $\mathbf{A}$. The inverse of $\mathbf{A 2 2}$ and its products with $\mathbf{S}$ matrices were computed using LAPACK subroutine DGEMM prior iterations and stored on disk. The SNP co-variance matrix was calculated as B $=\left[\sum_{j=1}^{m} 2 p_{j}\left(1-p_{j}\right)\right]^{-1} \mathbf{I}$, where $\mathbf{I}$ is an identity matrix. 


\section{Preconditioners}

The mixed model equations were solved by PCG method (Barrett et al., 1994). The system [2], written as $\mathbf{C x}=\mathbf{b}$, can be transformed to an equivalent system by choosing a preconditioner matrix $\mathbf{M}$ such that the preconditioned coefficient matrix $\mathbf{M}^{-1} \mathbf{C x}=\mathbf{M}^{-1} \mathbf{b}$ has a smaller spectral condition number than that of C. It should be pointed out the rate of convergence depends on the choice of M. Following the work of Frank and Vuik (2001), Vandenplas et al. (2018) proposed the use of second level preconditioner, called the deflation matrix, to achieve faster convergence of PCG. However, the system [2] is too large and the computation of the required deflation-subspace matrix becomes impractical.

In this study 2 types of preconditioners were used. The first one is the widely used block-diagonal preconditioner. For the fixed part of [2], the blocks of $\mathbf{M}$ are due to traits resulting in dense $t \times t$ blocks with $t$ being the number of traits. For the random part, the block size is equal to the dimension of the genetic co-variance matrix $\mathbf{G}_{0}$ or the permanent environment co-variance matrix $\mathbf{G}_{1}$. Therefore, $\mathbf{M}$ is a block-diagonal matrix with variable bock sizes. We introduced a second preconditioner for the SNP part of [2]. For the construction of such preconditioner we need the inverse of $\mathbf{G}_{0}^{-1} \otimes\left(\frac{1}{1-k} \mathbf{B}^{-1}+\frac{1}{k} \mathbf{S}^{\prime} \mathbf{A}_{22}^{-1} \mathbf{S}\right)$. Direct inverse is impossible given the size of the matrix. Using the rules of matrix inversion of Kronecker products, however, alleviates the problem. For square nonsingular matrices $\mathbf{A}$ and $\mathbf{B},(\mathbf{A} \otimes \mathbf{B})^{-1}=\mathbf{A}^{-1} \otimes \mathbf{B}^{-1}$. Then the multiplication of the right hand side by vector in PCG is achieved by using the vec trick.

In this study, $c^{n}=\frac{\left\|b-\mathbf{A} \mathbf{x}^{n}\right\|}{\|b\|}$ was used as a termination criteria, where $\|b\|$ is the Euclidian norm of $b$ from a linear system $\mathbf{A x}=b, n$ is the $n$th round of iteration, A represents the coefficient matrix of the linear system, $\mathbf{x}^{n}$ is the vector of solutions at round $n$, and $c^{n}$ is the relative difference between left-hand side and righthand side of the system of equations. The PCG was run until the termination criteria was less than or equal to $10^{-10}$.

\section{Calculation of Predictive Ability of sSSNP-BLUP}

For the validation of GEBV, the EBV of the official 305-d lactation total yields of milk, fat, and protein were obtained from the full data analysis without ge- nomics. For SCS the average of the 3 lactations was used. For fertility, only calving interval was considered. These EBV were de-regressed for bulls and cows. The expected daughter contributions (EDC) used as weights in the de-regression were calculated for cows with records only, free of contributions from relatives. The 5 traits were de-regressed separately using the official heritabilities supplied to Interbull: $h_{\text {milk }}^{2}=0.45$; $h_{\text {fat }}^{2}=0.50 ; h_{\text {protein }}^{2}=0.45 ; h_{S C S}^{2}=0.11 ; h_{\text {fertility }}^{2}=$ 0.036 .

The coefficient of determination $\left(\mathrm{R}^{2}\right)$ and the genomic prediction bias were obtained from the following linear regression equation:

$$
\mathrm{DRP}=b_{0}+b_{1} \mathrm{GEBV}+e,
$$

where DRP $=$ de-regressed proofs of bulls or cows in the full data sets, without genomics; GEBV is the genomic prediction based on the reduced data sets; $b_{0}$ and $b_{1}$ are unknown regression coefficients; and $e$ is a residual error. Similar tests were also applied to the PA. For each trait 2 separate analyses were performed: (1) analysis using genotype matrix $\mathbf{S}$ from the custom XT SNP list, and (2) analysis using genotype matrix $\mathbf{S}$ from the 50K Illumina chip.

\section{RESULTS AND DISCUSSION}

\section{Computational Requirement}

All analyses were performed on a server with 754 GB memory and $2 \times$ Intel (Intel, Santa Clara, CA) Xeon Platinum $8168 \mathrm{CPU} @ 2.70 \mathrm{GHz}$ with 48 cores in total. The feasibility of ssSNP-BLUP model was evaluated recording the number of iterations to reach convergence, the CPU time per iteration, and overall time for each trait analyzed. The results are shown in Table 3. Convergence issues with PCG method applied to ssSNP-BLUP have been reported in the literature (Taskinen et al., 2017; Vandenplas et al., 2018). We also experienced problems with convergence in our initial analyses when using the traditional block-diagonal preconditioner. It is well known that the convergence rate of the conjugate gradient method is bounded as a function of the condition number $k(C)$ of the system matrix to which it is applied (Frank and Vuik, 2001). Thus, the preconditioner matrix $\mathbf{M}$ must be chosen such that $\mathbf{M}^{-1} \mathbf{C} \leq k(C)$. Vandenplas et al. (2018) pointed out that in addition to the $k(C)$, other factors can affect the convergence rate such as the clustering of the eigenvalues of the system matrix, floating point round errors, and the right-hand side of the system. 
Table 3. Computing performance at convergence for traditional BLUP and single-step (ss)SNP-BLUP evaluations

\begin{tabular}{llccr}
\hline Model & $\begin{array}{l}\text { Type of } \\
\text { preconditioner }\end{array}$ & $\begin{array}{c}\text { Number of } \\
\text { iterations }\end{array}$ & $\begin{array}{c}\text { Time per } \\
\text { iteration (s) }\end{array}$ & $\begin{array}{c}\text { Total } \\
\text { time (h) }\end{array}$ \\
\hline $\begin{array}{l}\text { Traditional BLUP } \\
\text { MY, FY, PY }\end{array}$ & BDP & 1,360 & 123 & 46.5 \\
SCS & BDP & 1,268 & 44 & 15.5 \\
Cow fertility & BDP & 1,077 & 15 & 4.5 \\
SSSNP-BLUP & BDP & 1,516 & 139 & 58.5 \\
MY, FY, PY & SBDP & 1,040 & 152 & 43.9 \\
SCS & BDP & 1,225 & 48 & 16.3 \\
& SBDP & 760 & 54 & 11.4 \\
Cow fertility & BDP & 1,185 & 17 & 5.6 \\
& SBDP & 723 & 22 & 4.4
\end{tabular}

${ }^{1}$ Type of preconditioner: $\mathrm{BDP}=$ traditional block-diagonal; SBDP $=$ traditional block-diagonal plus second preconditioner.

${ }^{2} \mathrm{MY}=$ milk yield $; \mathrm{FY}=$ fat yield; and $\mathrm{PY}=$ protein yield.

The results in Table 3 show that the use of the second preconditioner resulted in a substantially smaller number of iterations to reach the termination criteria, compared with those obtained by using the traditional block-diagonal preconditioner. However, with very large matrices the construction of a second preconditioner using matrix inversion may not be feasible. The dimension of the matrices involved in the calculation of the preconditioner could be reduced by performing principal component analysis on the matrix of genotypes and subsequent rank reduction as was suggested by $\varnothing$ degård et al. (2018). This approach may also be challenging because the calculation of eigen values and vectors of very large matrices could be prohibitive. Another option that avoids inversion of a dense matrix could be to use an incomplete Cholesky or incomplete LU factorization of the SNP by SNP matrix, or construct a sparse approximate inverse preconditioner for a dense linear system. The construction of deflated preconditioners (Vandenplas et al., 2018) is worth investigating.

Utilizing genomic information for large-scale routine evaluations increased the memory requirements compared with the traditional BLUP evaluations. In this study we calculated several $\mathbf{A}_{22}^{-1}, \quad \mathbf{A}_{22}^{-1} \mathbf{S}$, and $\mathbf{S}^{\prime} \mathbf{A}_{22}^{-1} \mathbf{S}$ matrices for each breed with sizes $17,12.4$, and 14.4 GB, respectively. The corresponding time in seconds was $114.8,382.2$, and 394.4 , respectively. The memory and time also increased for the calculation of both preconditioners. For BDP we calculated 40,850 additional blocks of size $27 \times 27$ for each breed. For the second preconditioner we had to invert and retain $3 \mathbf{S}^{\prime} \mathbf{A}_{22}^{-1} \mathbf{S}$ matrices, thus increasing the memory requirements by another $44 \mathrm{~GB}$. The time required for the calculation of preconditioners was approximately $5.1 \mathrm{~h}$.

In the present study, the inverse of $\mathbf{A 2 2}$ was computed explicitly. The reason for this was that for matri- ces with moderate dimensions the inverse can be easily computed as Schur complement by using sparse matrix solvers from PARDISO. Our experience showed that using PARDISO for $50 \mathrm{~K}$ problem, the computation of the inverse of $\mathbf{A 2 2}$ takes 347.2 s with the use of 20 cores. For matrices with dimensions of $100 \mathrm{~K}, 150 \mathrm{~K}$, and $200 \mathrm{~K}$, the corresponding time in seconds was 2,386 , 6,542 , and 14,285 , respectively. It was suggested in the literature (Masuda et al., 2017; Strandén et al., 2017) that the computation of $\mathbf{A 2 2}$ inverse can be avoided using a sequence of sparse matrix-vector multiplications. However, the computation of the diagonal elements of the inverse of $\mathbf{A 2 2}$ may still be needed for the construction of appropriate preconditioners.

It is important to notice that the version of the program used is this study was not fully optimized. Different numbers of threads were used in the calculation of Kronecker product matrix by vector, but this part was still the most time consuming. Further improvements may reduce the computational time, such as the use of MPI libraries for more efficient parallel computing especially iteration on data part and Kronecker product matrix by vector. Multithreading computation of the preconditioners is also desirable. Another optimization could be the development of new more efficient preconditioners. Optimizations to reduce the computational time are required to allow weekly evaluations with ssSNP-BLUP.

\section{Predictive Ability of ssSNP-BLUP Model}

In the literature the validation reliabilities were calculated as the square of the Pearson correlation coefficient between GEBV or EBV and DRP (Gao et al., 2018; Oliveira et al., 2019), or as $\mathrm{R}^{2}$ of model [3] weighted by the average reliability of DRP (Mäntysaari 
et al., 2010; Koivula et al., 2015). It should be noted that the accuracy of the second method depends on the accuracy of the calculated EDC in the regression procedure. Calus et al. (2016) presented comprehensive review on the subject. In this study we reported the $\mathrm{R}^{2}$ from model [3] as a proxy to the validation reliability for each trait.

The validation reliabilities as well as the genomic prediction bias for Holstein, Jersey, and Red bulls and cows from analysis using the custom XT SNP list and 50K chip are shown in Tables 4, 5, and 6, respectively.

Predictions from evaluations based on phenotype and genomic information are expected to have higher $\mathrm{R}^{2}$ than traditional BLUP evaluations. For all traits and breeds, the $\mathrm{R}^{2}$ values from ssSNP-BLUP evaluations were higher compared with the PA values. Optimal predictions for bulls and cows should have regression coefficients of 1 . Both PA and GEBV were inflated or deflated. Generally, GEBV were less biased than PA for all traits and breeds. The regression coefficients were within the acceptable $\pm 15 \%$ deviation from unity (Tsuruta et al., 2011).

Lourenco et al. (2014) reported considerably lower $\mathrm{R}^{2}$ values for milk, fat, and protein yield GEBV obtained from ssGBLUP model in Israeli Holstein bulls. Our validation results for Holstein and Jersey bulls, for milk, fat, and protein yield are comparable to those reported by Wiggans et al. (2011) for US Holstein and Jersey bulls. Oliveira et al. (2019) used ssGBLUP random regression model for evaluation of Canadian Holstein Jersey and Ayrshire bulls for milk, fat, and protein yield, and SCS. Their validation reliabilities for milk, fat, and protein yield, and SCS in Holstein and Jersey breeds were similar to ours. However, for Ayrshire bulls their validation reliabilities were higher compared with ours for the Australian Red breed. For Nordic Red dairy breed, Koivula et al. (2015) also reported higher validation results for both bulls and cows evaluated using ssGBLUP multiple trait random regression model. Single-step GBLUP multitrait random regression model with different weighting parameters for the $\mathbf{H}$ matrix was used by Kang et al. (2018) for evaluation of Chinese Holsteins. Their validation results for milk and protein yield were slightly lower than ours but less biased for both bulls and cows. For Japanese Holstein bulls and cows, Baba et al. (2017) reported $\mathrm{R}^{2}$ ranged from 0.41 to 0.46 for milk yield. The lowest validation $R^{2}$ were found for cow fertility. Matilainen et al. (2018) reported considerably higher validation reliabilities for Nordic Red dairy cattle. For the Nordic Holstein population, Gao et al. (2012) reported validation reliabilities similar to ours for milk fat and protein yield but higher for fertility. In another study, Gao et al. (2018) presented validation reliabilities for Finnish

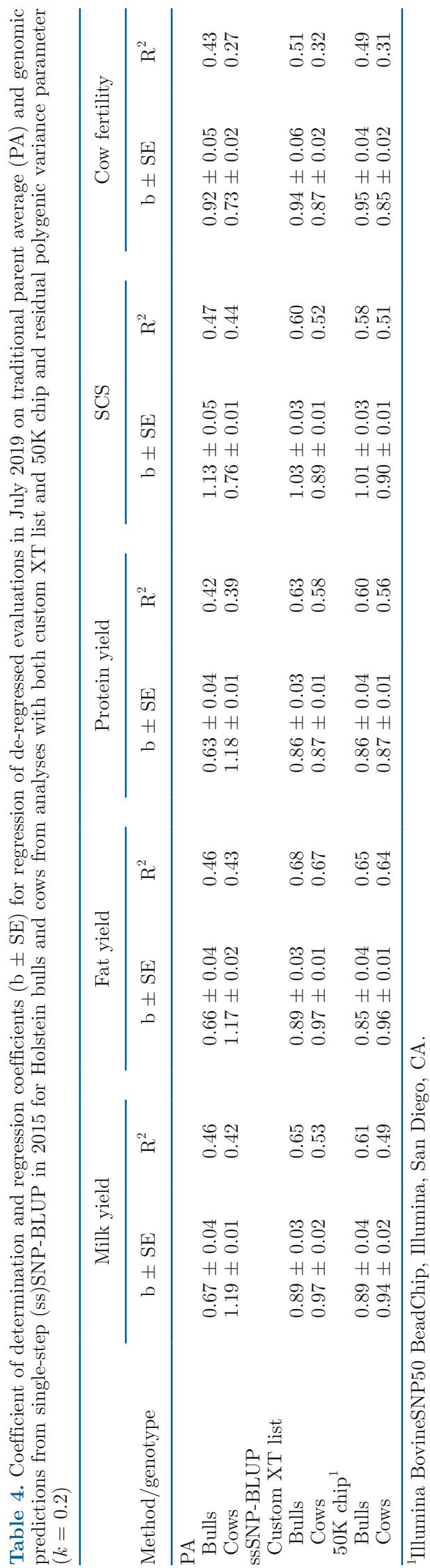


Konstantinov and Goddard: APPLICATION OF MULTIVARIATE SINGLE-STEP SNP-BLUP MODEL

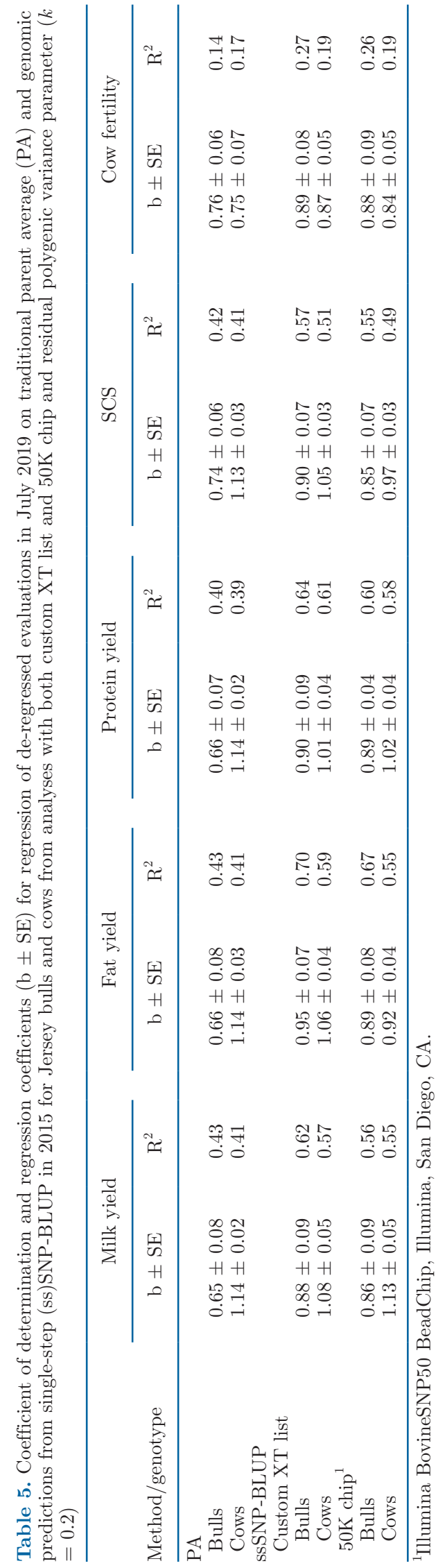

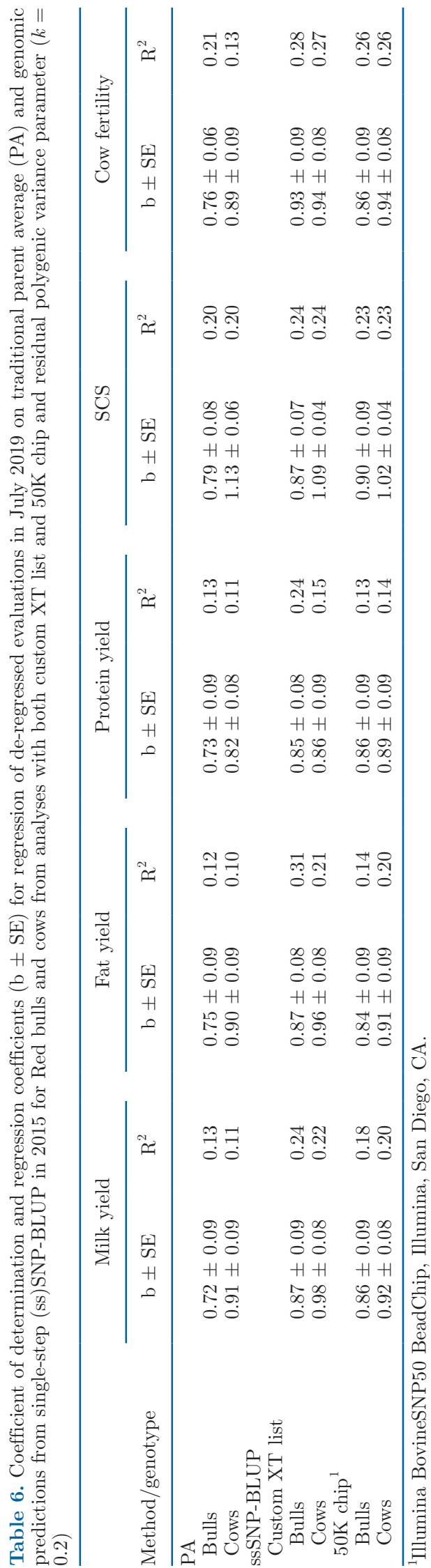


Red dairy cattle for milk fat and protein yield. Those results were in line with ours for the Australian Red cattle. The results from analysis using $50 \mathrm{~K}$ chip showed that the $\mathrm{R}^{2}$ values were also higher compared with the PA for all traits and breeds but lower compared with those obtained from the analysis with the custom XT list. The average percent increase in $\mathrm{R}^{2}$ from custom $\mathrm{XT}$ list compared with the $50 \mathrm{~K}$ chip for milk, fat, and protein yield varied from $5.3 \%$ to $7.8 \%$ for bulls and $3.6 \%$ to $7.7 \%$ for cows. Smaller increases were observed for SCS and fertility ranging from $3.5 \%$ to $5.5 \%$ for bulls and from $2.2 \%$ to $4.8 \%$ for cows. One explanation to this could be that the use of the custom XT SNP list resulted in better estimation of the SNP effects because the selected variants are in stronger linkage disequilibrium with causal variants, which in turn improved the predictive accuracy of the method.

The results from the analyses using the custom XT SNP list and different residual polygenic variance parameters are presented in Supplemental Tables S1, S2, S3, and S4 (https://doi.org/10.3168/jds.2020-18242). For bulls in Holstein and Jersey breed the $\mathrm{R}^{2}$ values decreased with the increase of the polygenic variance parameter from 0.2 to 0.8 . In Holstein breed the average decrease in $\mathrm{R}^{2}$ for milk, fat, and protein yield was $2.8,2.7$, and 4.7 percentage units, respectively. For Jersey the decrease was $2.8,3.9$, and 5.2 percentage units for milk, fat, and protein yield, respectively. For SCS and fertility, the decrease was small, around 1.5 percentage units. The opposite trend was observed in the Red breeds with the increase of the polygenic variance parameter. The trend in the regression coefficients was the same for most of the cases with the exception of $k=0.8$. There was a slight increase of the standard errors of the regression coefficients associated with the increase of the polygenic variance parameter. The trend in $\mathrm{R}^{2}$ with different values of the $\mathrm{k}$ parameter in this study was different from that found by Osawa and Saburi (2018) with $k=0.2,0.3,0.4$, and 0.5. They found that the lowest $\mathrm{R}^{2}$ value was with $k=0.2$. For the young bulls they found a similar decrease in the validation results with the increase of the $k$ parameter. However, they used the 305-d repeatability model for the estimation of the breeding values. For the cows in all breeds the decrease in $\mathrm{R}^{2}$ started from the use of $k$ $=0.4$ downward. The average value in percentage units seldom was above $1 \%$. A similar trend in $\mathrm{R}^{2}$ values and slopes was observed when the $50 \mathrm{~K}$ chip and different $k$ parameters were used in the analyses.

Liu et al. (2011) also observed small change in Pearson correlations of de-regressed EBV with GEBV for validation bulls when the percentage of residual polygenic variance varied from $5 \%, 10 \%$, and $20 \%$. One possible explanation could be that different percentage of residual polygenic variance have a small effect on SNP effect estimates (Liu et al., 2011). Those authors found that the variance of DGV defined as the sum of marker effects and the estimate of the residual polygenic effect remained constant across different values of $k$, indicating that the polygenic effect compensated for decreasing SNP effect.

For all traits and breeds, the ssSNP-BLUP yields higher validation reliabilities compared with the model without genomic information. For milk, fat, and protein yield and SCS, our validation results are in line with most of the ssGBLUP application studies available in the literature.

Our validation results for cows were somewhat higher compared with those reported in the literature, especially in the Jersey breed. A possible contributor to this result could be the way DRP were calculated. Calus et al. (2016) discussed in depth the performance of different de-regression procedures and pointed out that proper de-regression is more important for cows because they have lower reliabilities compared with bulls that have generally much higher reliabilities. The inclusion of information of both reference and validation animals in the calculation of DRP may lead to overestimation of the accuracy. The genetic relationship among bulls and among cows and among bulls and cows in the reference population could also affect the prediction accuracy in genomic evaluation. To obtain the highest accuracy of the validations reliabilities, additional research on the performance of different de-regression procedures is required.

For Red breeds, the validation results were lower than those reported in the literature. One possible explanation is that the Australian Red is quite a heterogeneous population. It combines extensive infusion of Scandinavian genetics with other Australian Red breeds such as Dairy Shorthorn, Ayrshire, Illawarra, or Red and White Holstein. In addition, the number of reference and validation animals may not have been sufficient.

The use of the custom XT SNP list resulted in better predictive ability compared with the validation results with the $50 \mathrm{~K}$ chip. This is likely because the SNP on the custom XT SNP list were selected to be more predictive than SNP on the 50K chip for a wide range of traits in Australian dairy cattle as described by Xiang et al. (2019). Also, approximately half of the genotyped animals used in our training set overlapped with animals used by Xiang and colleagues to select the custom XT SNP list. However, their phenotypes were generated from data available in 2016, whereas the current study used updated and new phenotypes available from the 2019 database. Several other studies have also reported improved accuracy of genomic prediction using sequence variants (Brøndum et al., 2015; van den 
Berg et al., 2016; VanRaden et al., 2017). Those studies generally reported smaller gains than ours; however, their approach was to add smaller sets of selected sequence variants to $50 \mathrm{~K}$ or HD SNP panels. In contrast, the majority of variants in the custom XT list were selected sequence variants, evenly spaced genome-wide, that were added to a low density set of approximately 8,000 SNP. Since completing this study, a custom XT array has been produced and several thousand animals of each breed have been genotyped with the chip to test direct imputation from the base set of 8,000 low-density SNP.

The use of different values of the polygenic residual variance parameter resulted in a slight decrease of $\mathrm{R}^{2}$ values. It should be pointed out that the problem of optimal partitioning of the additive genetic variance between the residual polygenic and SNP components is not resolved. Appropriate statistical methods are needed for the estimation of the residual polygenic variance.

The predictive ability of the ssSNP-BLUP method could be affected by several factors such as co-variance matrices, the use of phantom parent groups, and DRP. In the current study we used co-variance matrices estimated from models without genomics. These may not be the same as the ones estimated from ssSNP-BLUP model. However, with large commercial data sets this could be a challenging task. Another contributor could be the use of phantom parent groups. We deleted the groups from the relationship matrix for simplicity. Misztal et al. (2013) showed how the groups can be accommodated in the ssGBLUP model. Their approach is not directly applicable to ssSNP-BLUP. Therefore, one option for ssSNP-BLUP with genetic groups is to consider the groups as a separate factor of the model, similar to the models used in the early days of BLUP applications. The accuracy of the de-regressed proof could affect the validation results. In this study the DRP were calculated from "single" trait models. First, the daily EBV and heritabilities were converted to 305d EBV and heritabilities. In the case of cow fertility, only the calving interval was considered with the corresponding heritability. Strandén and Mäntysaari (2010) suggested the use of multivariate de-regression in cases where the EBV were calculated from multivariate models. However, their method requires modifications to accommodate models with heterogeneous residual covariances such as our test-day models.

\section{CONCLUSIONS}

Our results show that the use of ssSNP-BLUP to predict genomic breeding values for multitrait test-day records based on random regression models is feasible. The use of ssSNP-BLUP increased the reliability and reduced the bias of the breeding values compared with the PA from the traditional BLUP in the Australian Holstein, Jersey, and Red breeds. The use of the custom XT SNP list resulted in better predictive ability compared with the validation results with the $50 \mathrm{~K}$ chip.

\section{ACKNOWLEDGMENTS}

DataGene (Bundoora, VIC, Australia) is acknowledged for providing the data for analyses. We are grateful to Bolormaa Sunduimijid (Agriculture Victoria Research, AgriBio Centre, Bundoora, Australia) for genotype imputation and acknowledge our partners in the 1000 Bull Genomes Project for access to the reference genomes for imputation to sequence. We also thank Iona MacLeod (Agriculture Victoria Research, AgriBio Centre) for genotype processing. We thank the anonymous reviewers whose comments and suggestions helped improve and clarify this manuscript. The authors have not stated any conflicts of interest.

\section{REFERENCES}

Aguilar, I., I. Misztal, D. L. Johnson, A. Legarra, S. Tsuruta, and T. J. Lawlor. 2010. Hot topic: A unified approach to utilize phenotypic, full pedigree, and genomic information for genetic evaluation of Holstein final score. J. Dairy Sci. 93:743-752. https://doi.org/10 $.3168 /$ jds.2009-2730.

Baba, T., Y. Gotoh, S. Yamaguchi, S. Nakagawa, H. Abe, Y. Masuda, and T. Kawahara. 2017. Application of single-step genomic best linear unbiased prediction with a multiple-lactation random regression test-day model for Japanese Holsteins. Anim. Sci. J. 88:1226-1231. https://doi.org/10.1111/asj.12760.

Barrett, R., M. Berry, T. F. Chan, J. Demmel, J. M. Donato, J. Dongarra, V. Eijkhout, R. Pozo, C. Romine, and H. Van der Vorst. 1994. Templates for the solution of linear systems: Building blocks for iterative methods. Soc. Industrial Appl. Math. (SIAM), Philadelphia, PA. http://www.siam.org/books/.

Bouwman, A. C., H. D. Daetwyler, A. J. Chamberlain, C. H. Ponce, M. Sargolzaei, F. S. Schenkel, G. Sahana, A. Govignon-Gion, S. Boitard, M. Dolezal, H. Pausch, R. F. Brøndum, P. J. Bowman, B. Thomsen, B. Guldbrandtsen, M. S. Lund, B. Servin, D. J. Garrick, J. Reecy, J. Vilkki, A. Bagnato, M. Wang, J. L. Hoff, R. D. Schnabel, J. F. Taylor, A. A. E. Vinkhuyzen, F. Panitz, C. Bendixen, L. E. Holm, B. Gredler, C. Hozé, M. Boussaha, M. P. Sanchez, D. Rocha, A. Capitan, T. Tribout, A. Barbat, P. Croiseau, C. Drögemüller, V. Jagannathan, C. Vander Jagt, J. J. Crowley, A. Bieber, D. C. Purfield, D. P. Berry, R. Emmerling, K. U. Götz, M. Frischknecht, I. Russ, J. Sölkner, C. P. Van Tassell, R. Fries, P. Stothard, R. F. Veerkamp, D. Boichard, M. E. Goddard, and B. J. Hayes. 2018. Meta-analysis of genome-wide association studies for cattle stature identifies common genes that regulate body size in mammals. Nat. Genet. 50:362-367. https://doi.org/10.1038/ s41588-018-0056-5.

Brøndum, R. F., G. Su, L. Janss, G. Sahana, B. Guldbrandtsen, D. Boichard, and M. S. Lund. 2015. Quantitative trait loci markers derived from whole genome sequence data increases the reliability of genomic prediction. J. Dairy Sci. 98:4107-4116. https://doi.org/ 10.3168/jds.2014-9005

Calus, M. P. L., J. Vandenplas, J. Ten Napel, and R. F. Veerkamp. 2016. Validation of simultaneous deregression of cow and bull breeding values and derivation of appropriate weights. J. Dairy Sci. 99:6403-6419. https://doi.org/10.3168/jds.2016-11028. 
Christensen, O. F., and M. S. Lund. 2010. Genomic prediction when some animals are not genotyped. Genet. Sel. Evol. 42:2. https: //doi.org/10.1186/1297-9686-42-2 http://www.gsejournal.org/ content $/ 42 / 1 / 2$

Daetwyler, H. D., A. Capitan, H. Pausch, P. Stothard, R. van Binsbergen, R. F. Brøndum, X. Liao, A. Djari, S. C. Rodriguez, C. Grohs, D. Esquerré, O. Bouchez, M. N. Rossignol, C. Klopp, D. Rocha, S. Fritz, A. Eggen, P. J. Bowman, D. Coote, A. J. Chamberlain, C. Anderson, C. P. VanTassell, I. Hulsegge, M. E. Goddard, B. Guldbrandtsen, M. S. Lund, R. F. Veerkamp, D. A. Boichard, R. Fries, and B. J. Hayes. 2014. Whole-genome sequencing of 234 bulls facilitates mapping of monogenic and complex traits in cattle. Nat. Genet. 46:858-865. https://doi.org/10.1038/ng.3034.

Das, S., L. Forer, S. Schönherr, C. Sidore, A. E. Locke, A. Kwong, S. Vrieze, E. Y. Chew, S. Levy, M. McGue, D. Schlessinger, D. Stambolian, P. R. Loh, W. G. Iacono, A. Swaroop, L. J. Scott, F. Cucca, F. Kronenberg, M. Boehnke, G. R. Abecasis, and C. Fuchsberger. 2016. Next-generation genotype imputation service and methods. Nat. Genet. 48:1284-1287. https://doi.org/10.1038/ ng.3656.

Fernando, R. L., H. Cheng, B. L. Golden, and D. J. Garrick. 2016. Computational strategies for alternative single-step Bayesian regression models with large numbers of genotyped and non-genotyped animals. Genet. Sel. Evol. 48:96. https://doi.org/10.1186/ s12711-016-0273-2.

Fernando, R. L., J. C. M. Dekkers, and D. J. Garrick. 2014. A class of Bayesian methods to combine large numbers of genotyped and non-genotyped animals for whole-genome analyses. Genet. Sel. Evol. 46:50. https://doi.org/10.1186/1297-9686-46-50 http://www .gsejournal.org/content/46/50.

Frank, J., and C. Vuik. 2001. On the construction of deflation-based preconditioners. SIAM J. Sci. Comput. 23:442-462. https://doi .org/10.1137/S1064827500373231

Gao, H., O. F. Christensen, P. Madsen, U. S. Nielsen, Y. Zhang, M. S. Lund, and G. Su. 2012. Comparison on genomic predictions using three GBLUP methods and two single-step blending methods in the Nordic Holstein population. Genet. Sel. Evol. 44:8. https://doi .org/10.1186/1297-9686-44-8 http://www.gsejournal.org/content/ $44 / 1 / 8$.

Gao, H., M. Koivula, J. Jensen, I. Strandén, P. Madsen, T. Pitkänen, G. P. Aamand, and E. A. Mäntysaari. 2018. Short communication: Genomic prediction using different single-step methods in the Finnish red dairy cattle population. J. Dairy Sci. 101:10082-10088. https://doi.org/10.3168/jds.2018-14913.

Haile-Mariam, M., and J. E. Pryce. 2015. Variances and correlations of milk production, fertility, longevity, and type traits over time in Australian Holstein cattle. J. Dairy Sci. 98:7364-7379. https://doi .org/10.3168/jds.2015-9537.

Harris, B. L., and D. L. Johnson. 2010. Genomic predictions for New Zealand dairy bulls and integration with national genetic evaluation. J. Dairy Sci. 93:1243-1252. https://doi.org/10.3168/jds.2009 -2619 .

Hayes, B. J., P. J. Bowman, A. J. Chamberlain, and M. E. Goddard. 2009. Invited review: Genomic selection in dairy cattle: Progress and challenges. J. Dairy Sci. 92:433-443. https://doi.org/10.3168/ jds.2008-1646.

Henderson, H. V., and S. R. Searle. 1981. The vec-permutation matrix, the vec operator and Kronecker products: A review. Linear Multilinear Algebra 9:271-288. https://doi.org/10.1080/ 03081088108817379 .

Kang, H., C. Ning, L. Zhou, S. Zhang, Q. Yan, and J. F. Liu. 2018. Short communication: Single-step genomic evaluation of milk production traits using multiple-trait random regression model in Chinese Holsteins. J. Dairy Sci. 101:11143-11149. https://doi.org/ 10.3168/jds.2018-15090.

Koivula, M., I. Strandén, J. Pösö, G. P. Aamand, and E. A. Mäntysaari. 2015. Single step genomic evaluation using multitrait random regression model and test-day data. J. Dairy Sci. 98:2775-2784. https://doi.org/10.3168/jds.2014-8975.

Konstantinov, K. V., K. T. Beard, M. E. Goddard, and J. H. J. van der Werf. 2009. Genetic evaluation of Australian dairy cattle for somatic cell scores using multi-trait random regression test-day model. J. Anim. Breed. Genet. 126:209-215. https://doi.org/10 $.1111 /$ j.1439-0388.2008.00762.x.

Konstantinov, K. V., G. J. Nieuwhof, and T. P. Hancock. 2015. Implementation of Multiple traits Multi Lactation Random Regression Test Day Model for Production Traits in Australia. Interbull Bulletin No. 49. Orlando, Florida, July 9-12.

Legarra, A., I. Aguilar, and I. Misztal. 2009. A relationship matrix including full pedigree and genomic information. J. Dairy Sci. 92:4656-4663. https://doi.org/10.3168/jds.2009-2061.

Legarra, A., O. F. Christensen, Z. J. Vitezica, I. Aguilar, and I. Misztal. 2015. Ancestral relationships using metafounders: Finite ancestral populations and across population relationships. Genetics 200:455-468. https://doi.org/10.1534/genetics.115.177014.

Legarra, A., and V. Ducrocq. 2012. Computational strategies for national integration of phenotypic, genomic, and pedigree data in a single-step best linear unbiased prediction. J. Dairy Sci. 95:46294645. https://doi.org/10.3168/jds.2011-4982.

Liu, Z., M. E. Goddard, B. J. Hayes, F. Reinhardt, and R. Reents. 2016. Technical note: Equivalent genomic models with a residual polygenic effect. J. Dairy Sci. 99:2016-2025. https://doi.org/10 .3168/jds.2015-10394.

Liu, Z., M. E. Goddard, F. Reinhardt, and R. Reents. 2014. A singlestep genomic model with direct estimation of marker effects. J. Dairy Sci. 97:5833-5850. https://doi.org/10.3168/jds.2014-7924.

Liu, Z., F. R. Seefried, F. Reinhardt, S. Rensing, G. Thaller, and R. Reents. 2011. Impacts of both reference population size and inclusion of a residual polygenic effect on the accuracy of genomic prediction. Genet. Sel. Evol. 43:19. https://doi.org/10.1186/1297 -9686-43-19 http://www.gsejournal.org/contrnt/43/1/19.

Loh, P. R., P. Danecek, P. F. Palamara, C. Fuchsberger, A. Reshef, K. Finucane, S. Schoenherr, L. Forer, S. McCarthy, G. R. Abecasis, R. Durbin, and A. Price. 2016. Reference-based phasing using the Haplotype Reference Consortium panel. Nat. Genet. 48:14431448. https://doi.org/10.1038/ng.3679.

Lourenco, D. A. L., I. Misztal, S. Tsuruta, I. Aguilar, E. Ezra, M. Ron, A. Shirak, and J. I. Weller. 2014. Methods for genomic evaluation of a relatively small genotyped dairy population and effect of genotyped cow information in multiparity analyses. J. Dairy Sci. 97:1742-1752. https://doi.org/10.3168/jds.2013-6916.

Mäntysaari, E., Z. Liu, and P. VanRaden. 2010. Interbull validation test for genomic evaluations. Interbull Bull. 40:1-5.

Masuda, Y., I. Misztal, A. Legarra, S. Tsuruta, D. A. L. Lourenco, B. O. Fragomeni, and I. Aguilar. 2017. Technical note: Avoiding the direct inversion of the numerator relationship matrix for genotyped animals in single-step genomic best linear unbiased prediction solved with the preconditioned conjugate gradient. J. Anim. Sci. 95:49-52. https://doi.org/10.2527/jas.2016.0699.

Matilainen, K., I. Strandén, G. P. Aamand, and E. A. Mäntysaari. 2018. Single step genomic evaluation for female fertility in Nordic Red dairy cattle. J. Anim. Breed. Genet. 135:337-348. https://doi .org/10.1111/jbg.12353.

Misztal, I., Z. G. Vitezica, A. Legarra, I. Aguilar, and A. A. Swan. 2013. Unknown-parent groups in single-step genomic evaluation. J. Anim. Breed. Genet. 130:252-258. https://doi.org/10.1111/jbg .12025 .

Nieuwhof, G. J., K. T. Beard, K. V. Konstantinov, P. J. Bowman, and B. J. Hayes. 2010. Implementation of Genomics in Australia. Interbull Bulletin NO. 42. Riga, Latvia, May 31-June 4.

Ødegård, J., U. Indahl, I. Stranden, and T. Meuwissen. 2018. Largescale genomic prediction using singular value decomposition of the genotype matrix. Genet. Sel. Evol. 50:6. https://doi.org/10.1186/ s12711-018-0373-2.

Oliveira, H. R., D. A. Lourenco, Y. Masuda, I. Misztal, S. Tsuruta, J. Jamrozik, L. F. Brito, F. F. Silva, and F. S. Schenkel. 2019 Application of single-step genomic evaluation using multiple-trait random regression test-day models in dairy cattle. J. Dairy Sci. 102:2365-2377. https://doi.org/10.3168/jds.2018-15466.

Osawa, T., and J. Saburi. 2018. Study of single-step SNP BLUP in a Japanese Holstein population. Proceedings of the World Congress on Genetics Applied to Livestock Production, Auckland, New Zea- 
land. Electronic Poster Session-Methods and Tools-Models and Computing Strategies, volume 1, page 131.

Saad, Y. 2003. Iterative Methods for Sparse Linear Systems. 2nd ed. Society for Industrial and Applied Mathematics, Philadelphia, PA.

Sargolzaei, M., J. P. Chesnais, and F. S. Schenkel. 2014. A new approach for efficient genotype imputation using information from relatives. Genomics 15:478. http://www.biomedcentral.com/1471 $-2164 / 15 / 478$.

Sargolzaei, M., H. Iwaisaki, and J. J. Colleau. 2005. A fast algorithm for computing inbreeding coefficients in large populations. J. Anim. Breed. Genet. 122:325-331. https://doi.org/10.1111/j.1439 -0388.2005.00538.x.

Strandén, E. A., and E. A. Mäntysaari. 2010. A Recipe for Multiple Trait Deregression. Interbull Bulletin No. 42. Riga, Latvia, May 31-June 4, 2010.

Stranden, I., K. Matilainen, G. P. Aamand, and E. A. Mäntysaari. 2017. Solving efficiently large single-step genomic best linear unbiased prediction models. J. Anim. Breed. Genet. 134:264-274. https://doi.org/10.1111/jbg.12257.

Taskinen, M., E. A. Mäntysaari, and I. Strandén. 2017. Single-step SNP-BLUP with on-the-fly imputed genotypes and residual polygenic effects. Genet. Sel. Evol. 49:36. https://doi.org/10.1186/ s12711-017-0310-9.

Tsuruta, S., I. Misztal, I. Aguilar, and T. J. Lawlor. 2011. Multiple-trait genomic evaluation of linear type traits using genomic and phenotypic data in US Holsteins. J. Dairy Sci. 94:4198-4204. https://doi.org/10.3168/jds.2011-4256. van den Berg, I., D. Boichard, and M. S. Lund. 2016. Sequence variants selected from a multi-breed GWAS can improve the reliability of genomic predictions in dairy cattle. Genet. Sel. Evol. 48:83. https://doi.org/10.1186/s12711-016-0259-0.

Vandenplas, J., H. Eding, M. P. L. Calus, and C. Vuik. 2018. Defatted preconditioned conjugate gradient method for solving single-step BLUP models efficiently. Genet. Sel. Evol. 50:51. https://doi.org/ 10.1186/s12711-018-0429-3.

VanRaden, P. M. 2008. Efficient methods to compute genomic predictions. J. Dairy Sci. 91:4414-4423. https://doi.org/10.3168/jds .2007-0980.

VanRaden, P. M., M. E. Tooker, J. R. O'Connell, J. B. Cole, and D. M. Bickhart. 2017. Selecting sequence variants to improve genomic predictions for dairy cattle. Genet. Sel. Evol. 49:32. https://doi .org/10.1186/s12711-017-0307-4.

Wiggans, G. R., P. M. VanRaden, and T. A. Cooper. 2011. The genomic evaluation system in the United States: Past, present, future. J. Dairy Sci. 94:3202-3211. https://doi.org/10.3168/jds.2010 $-3866$.

Xiang, R., I. Berg, I. M. MacLeod, B. J. Hayes, C. P. Prowse-Wilkins, M. Wang, S. Bolormaa, Z. Liu, S. J. Rochfort, C. M. Reich, B. A. Mason, C. J. Vander Jagt, H. D. Daetwyler, M. S. Lund, A J. Chamberlain, and M. E. Goddard. 2019. Quantifying the contribution of sequence variants with regulatory and evolutionary significance to 34 bovine complex traits. Proc. Natl. Acad. Sci. USA 116:19398-19408. https://doi.org/10.1073/pnas.1904159116. 